TRANSACTIONS OF THE

AMERICAN MATHEMATICAL SOCIETY

Volume 356, Number 11, Pages 4427-4445

S 0002-9947(04)03386-0

Article electronically published on February 4, 2004

\title{
THE LOSS OF TIGHTNESS OF TIME DISTRIBUTIONS FOR HOMEOMORPHISMS OF THE CIRCLE
}

\author{
ZAQUEU COELHO
}

\begin{abstract}
For a minimal circle homeomorphism $f$ we study convergence in law of rescaled hitting time point process of an interval of length $\varepsilon>0$. Although the point process in the natural time scale never converges in law, we study all possible limits under a subsequence. The new feature is the fact that, for rotation numbers of unbounded type, there is a sequence $\varepsilon_{n}$ going to zero exhibiting coexistence of two non-trivial asymptotic limit point processes depending on the choice of time scales used when rescaling the point process. The phenomenon of loss of tightness of the first hitting time distribution is an indication of this coexistence behaviour. Moreover, tightness occurs if and only if the rotation number is of bounded type. Therefore tightness of time distributions is an intrinsic property of badly approximable irrational rotation numbers.
\end{abstract}

\section{INTRODUCTION}

Let $f$ be a minimal orientation preserving homeomorphism of the circle $S^{1}=$ $\mathbb{R} / \mathbb{Z}$ with rotation number $\alpha$. Let $\mu$ be the unique invariant Borel probability measure of $f$. Fix a point $z \in S^{1}$ and consider the interval $\mathfrak{I}_{\varepsilon}=[z, z+\varepsilon] \subseteq S^{1}$. For $\omega \in S^{1}$ define the first hitting time of $\mathfrak{I}_{\varepsilon}$ by

$$
\tau_{\varepsilon}(\omega)=\inf \left\{k>0: f^{k}(\omega) \in \mathfrak{I}_{\varepsilon}\right\} .
$$

The restriction of $\tau_{\varepsilon}$ to $\mathfrak{I}_{\varepsilon}$ is called the first return time of $\mathfrak{I}_{\varepsilon}$. The problem we address here is whether $\tau_{\varepsilon}$ when suitably rescaled converge in law when $\varepsilon$ tends to zero. Due to Kac's Lemma, which states that conditionally to starting at $\mathfrak{I}_{\varepsilon}$ the expected return time is $1 / \mu\left(\mathfrak{I}_{\varepsilon}\right)$, the natural time scale to be used for this problem is $\gamma_{\varepsilon}=\mu\left(\mathfrak{I}_{\varepsilon}\right)$. We are interested in the convergence of the distribution function of the random variable $X_{\varepsilon}(\cdot)=\mu\left(\mathfrak{I}_{\varepsilon}\right) \tau_{\varepsilon}(\cdot)$, i.e. in the convergence of the non-negative function

$$
F_{\varepsilon}(t)=\mu\left\{\omega \in S^{1}: \mu\left(\mathfrak{I}_{\varepsilon}\right) \tau_{\varepsilon}(\omega) \leq t\right\},
$$

as $\varepsilon \rightarrow 0$, for every $t$ belonging to the continuity points of the limit function. When $\varepsilon=\varepsilon_{n}$ is chosen such that $\mathfrak{I}_{\varepsilon_{n}}$ corresponds to a sequence of renormalisation intervals for $f$ as done in $[\mathrm{CF}$, it is proved in $[\mathrm{CF}]$ that for Lebesgue almost every rotation number $\alpha$, the rescaled hitting times $X_{\varepsilon}(\cdot)=\mu\left(\mathfrak{I}_{\varepsilon}\right) \tau_{\varepsilon}(\cdot)$ do not converge in law as $\varepsilon$ tends to zero, and all possible limit laws under a subsequence of $\varepsilon_{n}$ are obtained. Here we complement this statement by proving the following theorem.

Received by the editors August 31, 2001 and, in revised form, May 8, 2003.

2000 Mathematics Subject Classification. Primary 37E05, 11A55, 37A50; Secondary 28D05, $60 \mathrm{G} 55$.

Key words and phrases. Rotation numbers, limit laws, point processes, maps of the circle. 
Theorem 1. For any irrational rotation number $\alpha$, the rescaled hitting times $X_{\varepsilon}(\cdot)=\mu\left(\mathfrak{I}_{\varepsilon}\right) \tau_{\varepsilon}(\cdot)$ do not converge in law as $\varepsilon$ tends to zero.

We prove the above result by studying all the possible limit laws under a subsequence $\varepsilon_{n}$ of $\varepsilon \rightarrow 0$. In the process of proving Theorem 1 we obtain the following unusual phenomenon. Let $I_{n}$ be the interval with endpoints $\left\{z, f^{q_{n}}(z)\right\}$ contained in the same connected component of $S^{1} \backslash\{z, f(z)\}$, where $p_{n} / q_{n}$ are the sequence of rational convergents of $\alpha$.

Theorem 2. Let $\alpha$ be an irrational rotation number with continued fraction expansion $\left[a_{1}, a_{2}, \cdots\right]$ such that $\left\{a_{j}\right\}_{j \geq 1}$ is unbounded. Let $0<c<1$ be any fixed real number. If $a_{j_{n}} \rightarrow \infty$ take $\varepsilon_{n}$ such that $\mu\left(\mathfrak{I}_{\varepsilon_{n}}\right)=c \mu\left(I_{j_{n}-1}\right)$. The distribution functions $F_{\varepsilon_{n}}(t)$ of $X_{\varepsilon_{n}}(\cdot)=\mu\left(\mathfrak{I}_{\varepsilon_{n}}\right) \tau_{\varepsilon_{n}}(\cdot)$ converge uniformly on compact sets to the continuous piecewise linear function

$$
F_{\mu}(t)= \begin{cases}t & \text { if } 0 \leq t \leq c \\ c & \text { if } t>c\end{cases}
$$

and $F_{\mu}(t)=0$ if $t<0$. Therefore the distributions of $X_{\varepsilon_{n}}(\cdot)=\mu\left(\mathfrak{I}_{\varepsilon_{n}}\right) \tau_{\varepsilon_{n}}(\cdot)$ are not tight.

This means that in the natural time scale $\mu\left(\mathfrak{I}_{\mathcal{\varepsilon}_{n}}\right)$, there are subsets $A_{n}$ in the circle with measure bounded away from zero, whose points "asymptotically never" reach $\mathfrak{I}_{\varepsilon_{n}}$, i.e. there exists $N_{n} \rightarrow \infty$ such that $\mu\left\{\omega \in S^{1}: \mu\left(\mathfrak{I}_{\varepsilon_{n}}\right) \tau_{\varepsilon_{n}}(\omega)>N_{n}\right\} \rightarrow$ $1-c>0$.

Since Lebesgue almost every irrational number is not of bounded type, Theorem 2 implies that for Lebesgue almost every rotation number $\alpha$, the distributions of $X_{\varepsilon}(\cdot)=\mu\left(\mathfrak{I}_{\varepsilon}\right) \tau_{\varepsilon}(\cdot)$ are not tight. In fact, a stronger statement holds.

Theorem 3. The distributions of $X_{\varepsilon}(\cdot)=\mu\left(\mathfrak{I}_{\varepsilon}\right) \tau_{\varepsilon}(\cdot)$ are tight if and only if the rotation number $\alpha$ is of bounded type.

The above result shows that tightness of time distributions is an intrinsic property of badly approximable irrational rotation numbers. The loss of tightness may indicate in general the presence of another time scale $\gamma_{\varepsilon} \neq \mu\left(\mathfrak{I}_{\varepsilon}\right)$ with a different non-trivial limit law. This is indeed the case for circle homeomorphisms as the next result shows.

Theorem 4. Let $\alpha$ be an irrational rotation number with continued fraction expansion $\left[a_{1}, a_{2}, \cdots\right]$ such that $\left\{a_{j}\right\}_{j \geq 1}$ is unbounded. Let $0<c<1$ be any fixed real number. If $a_{j_{n}} \rightarrow \infty$ take $\varepsilon_{n}$ such that $\mu\left(\mathfrak{I}_{\varepsilon_{n}}\right)=c \mu\left(I_{j_{n}-1}\right)$ and define $\gamma_{\varepsilon_{n}}=\mu\left(I_{j_{n}}\right)$. The distribution functions $F_{\varepsilon_{n}}^{Y}(t)$ of $Y_{\varepsilon_{n}}(\cdot)=\gamma_{\varepsilon_{n}} \tau_{\varepsilon_{n}}(\cdot)$ converge pointwise to the limit function

$$
F_{\gamma}(t)= \begin{cases}t+c & \text { if } 0<t \leq 1-c ; \\ 1 & \text { if } t>1-c ;\end{cases}
$$

and $F_{\gamma}(t)=0$ if $t \leq 0$.

Theorems 2 and 4 show the coexistence of two non-trivial asymptotic time distributions for the same sequence of shrinking intervals $\mathfrak{I}_{\varepsilon_{n}}$; see Figure丩, Note that if $f$ is a rigid rotation, i.e. $f(x)=x+\alpha(\bmod 1)$, then $\mu\left(\mathfrak{I}_{\varepsilon_{n}}\right)=\varepsilon_{n}=c\left|q_{j_{n}-1} \alpha-p_{j_{n}-1}\right|$ and $\gamma_{\varepsilon_{n}}=\left|q_{j_{n}} \alpha-p_{j_{n}}\right|$. 

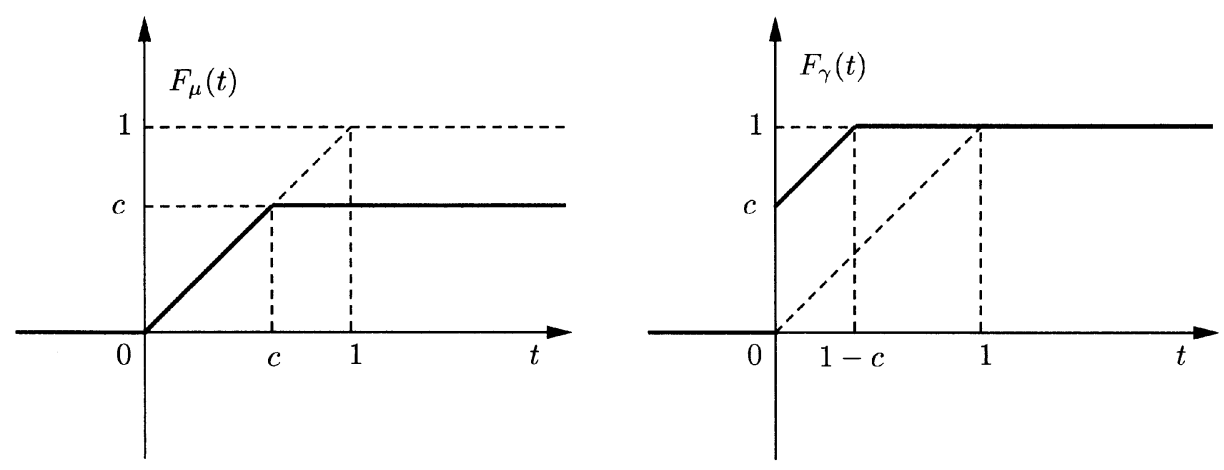

FIgURE 1. The coexistence behaviour:

$\mu\left\{\omega \in S^{1}: \mu\left(\mathfrak{I}_{\varepsilon_{n}}\right) \tau_{\varepsilon_{n}}(\omega) \leq t\right\} \rightarrow F_{\mu}(t)$,

$\mu\left\{\omega \in S^{1}: \gamma_{\varepsilon_{n}} \tau_{\varepsilon_{n}}(\omega) \leq t\right\} \rightarrow F_{\gamma}(t)$.

In Section 6 we show that convergence in law of the first hitting time under a subsequence $\varepsilon_{n} \rightarrow 0$ implies convergence in law of the full hitting time point process of $\mathfrak{I}_{\varepsilon_{n}}$ and we obtain all possible limit processes. Under the hypotheses of Theorems 2 and 4 we show the coexistence of two non-trivial independent limit point processes depending on the choice of scale $\gamma_{\varepsilon_{n}}$. Choosing $\gamma_{\varepsilon_{n}}=\mu\left(\mathfrak{I}_{\varepsilon_{n}}\right)$ the limit point process is degenerate giving probability $1-c$ to infinity or it is the lattice point process of increment $c$ with probability $c$. Choosing $\gamma_{\varepsilon_{n}}=\mu\left(I_{j_{n}}\right)$ the limit point process has no increment with probability one and the first hit is a mixed random variable with distribution given by $F_{\gamma}(t)$.

In Section 7 we complete the paper by studying the above questions when conditioning the hitting time point process to $\mathfrak{I}_{\varepsilon_{n}}$, i.e. the study of the return time point process of $\mathfrak{I}_{\varepsilon_{n}}$, and we show that the loss of tightness phenomenon does not occur for return times, and also there is no coexistence of two non-trivial point processes by choosing different time scales.

The results of this paper can be extended to diffeomorphisms of the circle which are $C^{1}$-conjugate to an irrational rotation and replacing the invariant measure $\mu$ by Lebesgue measure. This can be done in a similar way as done in Section 5 of $\mathrm{CF}$. The limit distributions will have a scale factor $g(z)$, where $g(z)$ is the density of the absolutely continuous invariant measure $\mu$.

We should mention that asymptotic time distributions have been obtained in a number of contexts, when studying hitting and return times of neighbourhoods of generic points in the natural scale of the measure of the neighbourhoods. For finite state Markov chains and Anosov diffeomorphisms [Pit, Axiom A diffeomorphisms [Hir], piecewise expanding maps of the interval [CG] and non-uniformly hyperbolic interval maps with indifferent fixed point [HSV] (the finite measure case), the limit laws of rescaled hitting times of shrinking neighbourhoods of a generic point are all exponential of parameter one. This universal behaviour do not hold for low complexity systems, since $[\mathrm{CF}$ and the present paper show that for irrational rotations the limit law does not exist. In $[\overline{D M}]$ the authors study this problem for a class of low complexity Cantor minimal systems (which contains a class of substitution systems) and they obtain piecewise linear limit laws with many branches and slopes. 
The loss of tightness appeared in [DM] when they considered the odometer, however they did not study another scale showing coexistence of different asymptotic limit laws. For non-uniformly hyperbolic interval maps with indifferent fixed point (the infinite measure case), the limit law is the independent product of the power of an exponential law and an $\alpha$-stable law; see [BZ].

\section{INDUCING ON RENORMALISATION INTERVALS}

Let $G:[0,1) \rightarrow[0,1)$ denote the Gauss transformation $G(x)=1 / x \bmod 1$ if $x>0$ and define $G(0)=0$. We also introduce the double Gauss transformation $\Gamma:[0,1)^{2} \rightarrow[0,1)^{2}$ given by

$$
\Gamma(\alpha, \beta)=\left(G(\alpha), \frac{1}{a(\alpha)+\beta}\right)
$$

where $a(x)$ denotes the integer part of $1 / x$ (i.e. the first digit of the continued fraction expansion of $x$ ). Here we are using the convention that $a(0)=\infty$ and $1 / \infty=0$. Note that for $n \geq 1$,

$$
\Gamma^{n}(\alpha, \beta)=\left(G^{n}(\alpha),\left[a_{n}, a_{n-1}, \cdots, a_{1}, b_{1}, b_{2}, \cdots\right]\right),
$$

where $a_{j}=a\left(G^{j-1}(\alpha)\right), b_{j}=a\left(G^{j-1}(\beta)\right)$, for $j \geq 1$, and hence the convergent subsequences of $\Gamma^{n}(\alpha, \beta)$ for $n \geq 0$ do not depend on $\beta$.

With the hypothesis of minimality, we recall that the homeomorphism $f$ is conjugate to the rigid rotation $R_{\alpha}(x)=x+\alpha$ mod 1 under the map $h:[0,1) \rightarrow[0,1)$ given by $h(x)=\mu[0, x)$, which carries the invariant measure $\mu$ to Lebesgue measure on $S^{1}$. Given the interval $\mathfrak{I}_{\varepsilon}=[z, z+\varepsilon)$ we consider the image $h\left(\mathfrak{I}_{\varepsilon}\right)=\left[h(z), h(z)+r_{z}(\varepsilon)\right)$. Note that for fixed $z$, the function $\varepsilon \mapsto r_{z}(\varepsilon)=h(z+\varepsilon)-h(z)$ is continuous and non-decreasing. Since hitting times and return times are preserved by the conjugacy $h$ and since $\mu\left(\mathfrak{I}_{\varepsilon}\right)=\left|h\left(\mathfrak{I}_{\varepsilon}\right)\right|$, where $|\cdot|$ stands for the length, we may assume without loss of generality that $f=R_{\alpha}$ and prove our results in this special case. Hence, in what follows we assume that $f$ is the rigid rotation by $\alpha$.

If the continued fraction expansion of $\alpha$ is given by $\left[a_{1}, a_{2}, \cdots\right]$, then define the rational convergents of $\alpha$ as the irreducible fraction $p_{n} / q_{n}$ given by

$$
\frac{p_{n}}{q_{n}}=\left[a_{1}, \cdots, a_{n}\right]=\frac{1}{a_{1}+\frac{1}{\cdots+\frac{1}{a_{n}}}} .
$$

The points $\{z, f(z)\}$ divide the circle into two connected components which we will refer to as the left and right side of $z$ with respect to the natural local ordering of the reals on $S^{1}=\mathbb{R} / \mathbb{Z}$. It is a well-known fact that $q_{n}$ is exactly the sequence of integers $k$ such that

$$
\left|z-f^{k}(z)\right|<\min _{0<j<k}\left|z-f^{j}(z)\right|
$$

and that the points $f^{q_{n-1}}(z)$ and $f^{q_{n}}(z)$ lie on opposite sides of $z$. Let $I_{n}$ be the closed interval with endpoints $\left\{z, f^{q_{n}}(z)\right\}$, whose interior points are on the same side with respect to $z$. Define $J_{n}=I_{n} \cup I_{n-1}$. Hence $z \in J_{n}$ and $I_{n} \cap I_{n-1}=\{z\}$. The intervals $I_{n}$ and $J_{n}$ are called renormalisation intervals for $f$.

Inducing $f$ on the interval $J_{n}$ defines the first return map $T=T_{n}: J_{n} \rightarrow J_{n}$ given by

$$
T(x)= \begin{cases}f^{q_{n}}(x) & \text { if } x \in I_{n-1} \backslash\{z\} \\ f^{q_{n-1}}(x) & \text { if } x \in I_{n}\end{cases}
$$


It is interesting to note that $f^{q_{n}+q_{n-1}}(z) \in I_{n-1}$ and hence $f^{q_{n-1}}$ maps $I_{n}$ onto a closed subinterval of $I_{n-1}$ of the same length of $I_{n}$ and with endpoints $\left\{f^{q_{n}+q_{n-1}}(z)\right.$, $\left.f^{q_{n-1}}(z)\right\}$. Similarly, $f^{q_{n}}$ maps $I_{n-1}$ onto a closed subinterval of $J_{n}$ with endpoints $\left\{f^{q_{n}}(z), f^{q_{n}+q_{n-1}}(z)\right\}$. Applying the return map $T$ repeatedly on $I_{n}$ we see that $T^{k+1}\left(I_{n}\right) \cap T^{k}\left(I_{n}\right)=\left\{f^{k q_{n}+q_{n-1}}(z)\right\}$, for $k=1, \cdots, a_{n}$. Since $q_{n+1}=a_{n} q_{n}+q_{n-1}$, we see that $a_{n}$ is the maximum number of copies of $I_{n}$ that one can fit in $I_{n-1}$ by repeated applications of $T$. From this we obtain $\left|I_{n+1}\right|+a_{n}\left|I_{n}\right|=\left|I_{n-1}\right|$. Therefore we have

$$
\frac{q_{n-1}}{q_{n}}=\frac{1}{a_{n-1}+\frac{1}{\cdots+\frac{1}{a_{1}}}} \text { and } \frac{\left|I_{n}\right|}{\left|I_{n-1}\right|}=\frac{1}{a_{n}+\frac{1}{a_{n+1}+\frac{1}{\cdots}}}
$$

hence we see that

$$
\left(\frac{\left|I_{n}\right|}{\left|I_{n-1}\right|}, \frac{q_{n-1}}{q_{n}}\right)=\Gamma^{n-1}(\alpha, 0) .
$$

Inducing $f$ on $I_{n-1}$ we obtain the map $S=S_{n}: I_{n-1} \rightarrow I_{n-1}$ given by

$$
S(x)= \begin{cases}f^{q_{n}}(x) & \text { if } x \in I_{n-1} \backslash T^{-1}\left(I_{n} \backslash\{z\}\right) \\ f^{q_{n}+q_{n-1}}(x) & \text { if } x \in T^{-1}\left(I_{n} \backslash\{z\}\right) .\end{cases}
$$

Let $\tau_{J}: S^{1} \rightarrow \mathbb{N}$ and $\tau_{I}: S^{1} \rightarrow \mathbb{N}$ denote the first hitting time of $J_{n}$ and $I_{n-1}$ respectively, i.e. for $\omega \in S^{1}$,

$$
\tau_{J}(\omega)=\inf \left\{k>0: f^{k}(\omega) \in J_{n}\right\} \quad \text { and } \quad \tau_{I}(\omega)=\inf \left\{k>0: f^{k}(\omega) \in I_{n-1}\right\} .
$$

Consider the rescaled first hitting time random variables defined by $X_{J}(\cdot)=$ $\left|J_{n}\right| \tau_{J}(\cdot)$ and $X_{I}(\cdot)=\left|I_{n-1}\right| \tau_{I}(\cdot)$, where $\left|J_{n}\right|$ and $\left|I_{n-1}\right|$ denote the length of the corresponding intervals. Let $F_{J}$ and $F_{I}$ denote the distribution functions of $X_{J}$ and $X_{I}$ respectively, i.e. for $t \in \mathbb{R}$,

$$
F_{J}(t)=\mu\left\{\omega \in S^{1}: X_{J}(\omega) \leq t\right\} \quad \text { and } \quad F_{I}(t)=\mu\left\{\omega \in S^{1}: X_{I}(\omega) \leq t\right\} .
$$

The collection of intervals $\left\{I_{n}, f\left(I_{n}\right), \cdots, f^{q_{n-1}-1}\left(I_{n}\right), I_{n-1}, f\left(I_{n-1}\right), \cdots\right.$, $\left.f^{q_{n}-1}\left(I_{n-1}\right)\right\}$ cover the circle and the intersection of any two of these intervals is either empty or consists of a single point. This fact implies that for $0<k \leq q_{n-1}$ we have

$$
\mu\left\{\omega \in S^{1}: \tau_{J}(\omega)=k\right\}=\left|I_{n}\right|+\left|I_{n-1}\right|=\left|J_{n}\right|,
$$

and for $q_{n-1}<k \leq q_{n}$,

$$
\mu\left\{\omega \in S^{1}: \tau_{J}(\omega)=k\right\}=\left|I_{n-1}\right| .
$$

Similarly, we have for $0<k \leq q_{n}$,

$$
\mu\left\{\omega \in S^{1}: \tau_{I}(\omega)=k\right\}=\left|I_{n-1} \backslash T^{-1}\left(I_{n}\right)\right|+\left|T^{-1}\left(I_{n}\right)\right|=\left|I_{n-1}\right|,
$$

and for $q_{n}<k \leq q_{n}+q_{n-1}$,

$$
\mu\left\{\omega \in S^{1}: \tau_{I}(\omega)=k\right\}=\left|T^{-1}\left(I_{n}\right)\right|=\left|I_{n}\right| .
$$


These observations readily give

Proposition 5. (a) For $J=J_{n}$ we have

$$
\begin{gathered}
F_{J}(t)=\left\{\begin{array}{lc}
k\left|J_{n}\right| & \text { if } k\left|J_{n}\right| \leq t<(k+1)\left|J_{n}\right|, \\
\text { and } 0 \leq k<q_{n-1} ; \\
\left(k-q_{n-1}\right)\left|I_{n-1}\right|+q_{n-1}\left|J_{n}\right| & \text { if } k\left|J_{n}\right| \leq t<(k+1)\left|J_{n}\right|, \\
\text { and } q_{n-1} \leq k<q_{n}
\end{array}\right.
\end{gathered}
$$

(b) For $I=I_{n-1}$ we have

$$
\begin{aligned}
& F_{I}(t)=\left\{\begin{array}{cc}
k\left|I_{n-1}\right| & \text { if } k\left|I_{n-1}\right| \leq t<(k+1)\left|I_{n-1}\right|, \\
& \text { and } 0 \leq k<q_{n} \\
\left(k-q_{n}\right)\left|I_{n}\right|+q_{n}\left|I_{n-1}\right| & \text { if } k\left|I_{n-1}\right| \leq t<(k+1)\left|I_{n-1}\right|, \\
& \text { and } q_{n} \leq k<q_{n}+q_{n-1} ;
\end{array}\right. \\
& F_{I}(t)=0 \text { for } t \leq 0 \text { and } F_{I}(t)=1 \text { for } t \geq\left(q_{n}+q_{n-1}\right)\left|I_{n-1}\right| \text {. }
\end{aligned}
$$

Let $n_{k}$ be a subsequence of $n \in \mathbb{N}$ and consider the distribution functions $F_{n_{k}}^{J}=F_{J_{n_{k}}}$ and $F_{n_{k}}^{I}=F_{I_{n_{k}-1}}$. By approximating by a continuous piecewise linear function (as done in $[\mathrm{CF}]$ ) we see that $F_{n_{k}}^{J}$ converges pointwise and uniformly if and only if the slope $\left|I_{n_{k}-1}\right| /\left|J_{n_{k}}\right|$ together with the endpoints $q_{n_{k}-1}\left|J_{n_{k}}\right|$ and $q_{n_{k}}\left|J_{n_{k}}\right|$ converges as $k \rightarrow \infty$. Similarly, $F_{n_{k}}^{I}$ converges pointwise and uniformly if and only if the slope $\left|I_{n_{k}}\right| /\left|I_{n_{k}-1}\right|$ together with the endpoints $q_{n_{k}}\left|I_{n_{k}-1}\right|$ and $\left(q_{n_{k}}+q_{n_{k}-1}\right)\left|I_{n_{k}-1}\right|$ converges as $k \rightarrow \infty$. The convergence occurs simultaneously for $F_{n_{k}}^{J}$ and $F_{n_{k}}^{I}$ and it happens if and only if either (a) $\left|I_{n_{k}}\right| /\left|I_{n_{k}-1}\right|$ converges to zero, which is equivalent to

$$
q_{n_{k}-1}\left|J_{n_{k}}\right| \rightarrow 0, q_{n_{k}}\left|J_{n_{k}}\right| \rightarrow 1, q_{n_{k}}\left|I_{n_{k}-1}\right| \rightarrow 0 \text { and }\left(q_{n_{k}}+q_{n_{k}-1}\right)\left|I_{n_{k}-1}\right| \rightarrow 1 ;
$$

or (b) $\left|I_{n_{k}}\right| /\left|I_{n_{k}-1}\right|$ converges to $\theta>0$ and $q_{n_{k}-1} / q_{n_{k}}$ converges to $\nu<1$. From (3) and (5), these conditions in terms of the Gauss map and the double Gauss map are stated as follows.

Proposition 6. Both $F_{n_{k}}^{J}$ and $F_{n_{k}}^{I}$ converge pointwise and uniformly if and only if either

(a) $\lim _{k \rightarrow \infty} G^{n_{k}-1}(\alpha)=0$, which is equivalent to $a_{n_{k}} \rightarrow \infty$; or

(b) there exist $\theta>0$ and $\nu<1$ such that $\lim _{k \rightarrow \infty} \Gamma^{n_{k}-1}(\alpha, \cdot) \rightarrow(\theta, \nu)$.

In case (a) both $F_{n_{k}}^{J}$ and $F_{n_{k}}^{I}$ converge to the uniform distribution in $[0,1]$. However, case (b) is equivalent to

$$
\lim _{k \rightarrow \infty} \frac{\left|I_{n_{k}}\right|}{\left|I_{n_{k}-1}\right|}=\theta \text { and } \lim _{k \rightarrow \infty} \frac{q_{n_{k}-1}}{q_{n_{k}}}=\nu .
$$

Therefore, using the identity $q_{n}\left|I_{n-1}\right|+q_{n-1}\left|I_{n}\right|=1$, which holds for all $n$, we have

and

$$
\lim _{k \rightarrow \infty} \frac{\left|I_{n_{k}-1}\right|}{\left|J_{n_{k}}\right|}=\frac{1}{1+\theta}, \quad \lim _{k \rightarrow \infty} q_{n_{k}-1}\left|J_{n_{k}}\right|=\frac{(1+\theta) \nu}{1+\theta \nu}
$$

$$
\lim _{k \rightarrow \infty} q_{n_{k}}\left|J_{n_{k}}\right|=\frac{1+\theta}{1+\theta \nu} .
$$


We also have

$$
\lim _{k \rightarrow \infty} q_{n_{k}}\left|I_{n_{k}-1}\right|=\frac{1}{1+\theta \nu} \quad \text { and } \quad \lim _{k \rightarrow \infty}\left(q_{n_{k}}+q_{n_{k}-1}\right)\left|I_{n_{k}-1}\right|=\frac{1+\nu}{1+\theta \nu} .
$$

Therefore, in case (b), $F_{n_{k}}^{J}$ and $F_{n_{k}}^{I}$ will converge to the same distribution function if and only if $\theta=\nu$ and $\theta(1+\theta)=1$, i.e. $\theta=(-1+\sqrt{5}) / 2=[1,1, \cdots]$ is the golden ratio.

Let $\tau_{\varepsilon}: S^{1} \rightarrow \mathbb{N}$ denote the first hitting time of $\mathfrak{I}_{\varepsilon}$, i.e. for $\omega \in S^{1}$,

$$
\tau_{\varepsilon}(\omega)=\inf \left\{k>0: f^{k}(\omega) \in \mathfrak{I}_{\varepsilon}\right\} .
$$

Consider the rescaled first hitting time random variables defined by $X_{\varepsilon}(\cdot)$ $=\left|\mathfrak{I}_{\varepsilon}\right| \tau_{\varepsilon}(\cdot)=\varepsilon \tau_{\varepsilon}(\cdot)$. Let $F_{\varepsilon}$ denote the distribution function of $X_{\varepsilon}$, i.e. for $t \in \mathbb{R}$,

$$
F_{\varepsilon}(t)=\mu\left\{\omega \in S^{1}: X_{\varepsilon}(\omega) \leq t\right\} .
$$

From the above considerations, taking subsequences $\varepsilon_{n_{k}}=\left|J_{n_{k}}\right|$ and $\varepsilon_{n_{k}}^{\prime}=$ $\left|I_{n_{k}-1}\right|$, we have proved the following result.

Lemma 7. If $X_{\varepsilon}$ converges in law as $\varepsilon \rightarrow 0$, then the rotation number $\alpha$ satisfies necessarily one of the following possibilities:

(a) $\lim _{n \rightarrow \infty} G^{n}(\alpha)=0$; or

(b) $\lim _{n \rightarrow \infty} G^{n}(\alpha)=[1,1, \cdots]$ is the golden ratio, i.e. there exists $N>0$ such that $G^{N}(\alpha)=[1,1, \cdots]$.

\section{INDUCING ON THE INTERVAL $\mathfrak{I}_{\varepsilon}$}

Here we take $\mathfrak{I}_{\varepsilon}=[z, z+\varepsilon]$ with $\varepsilon>0$ small enough such that there exists a unique $n>0$ such that $\left|I_{n}\right|<\varepsilon \leq\left|I_{n-1}\right|$. We wish to induce the map $f$ on the interval $\mathfrak{I}_{\varepsilon}$. We will show that the first return map $U=U_{\varepsilon}: \mathfrak{I}_{\varepsilon} \rightarrow \mathfrak{I}_{\varepsilon}$ is an interval exchange transformation of three subintervals of $\mathfrak{I}_{\varepsilon}$ with permutation (321), which is a classical fact 1 Note from the construction of the map $T$ that $f^{-q_{n}}(z)$ belongs to the interior of $\mathfrak{I}_{\varepsilon}$. Assume without loss of generality that (for this fixed $n$ )

$$
f^{q_{n}}(z)<z<f^{-q_{n}}(z)<z+\varepsilon<f^{q_{n-1}}(z) .
$$

Consider the interval $W_{\varepsilon}=\left[f^{-q_{n}}(z), z+\varepsilon\right]$. Since $f^{q_{n}}$ sends $W_{\varepsilon}$ to the interval of the same length $\left[z, f^{q_{n}}(z+\varepsilon)\right] \subset \mathfrak{I}_{\varepsilon}$, we have $U(x)=f^{q_{n}}(x)$ if $x \in W_{\varepsilon}$.

Let $a_{\varepsilon}$ with $1 \leq a_{\varepsilon} \leq a_{n}$ be defined as the smallest $k \geq 1$ such that $f^{k q_{n}+q_{n-1}}(z)$ belongs to the interior of $\mathfrak{I}_{\varepsilon}$. Note that this is the smallest $k \geq 1$ such that $T^{k}\left(I_{n}\right) \cap \mathfrak{I}_{\varepsilon}$ has non-empty interior. Define $q_{\varepsilon}=a_{\varepsilon} q_{n}+q_{n-1}$. Since $f^{-q_{\varepsilon}}(z+\varepsilon)$ belongs to $\left[z, f^{-q_{n}}(z)\right]$, the interval $\mathfrak{I}_{\varepsilon}$ is subdivided into the three intervals $K_{\varepsilon}=$ $\left[z, f^{-q_{\varepsilon}}(z+\varepsilon)\right], V_{\varepsilon}=\left[f^{-q_{\varepsilon}}(z+\varepsilon), f^{-q_{n}}(z)\right]$ and $W_{\varepsilon}$. However, $V_{\varepsilon}$ may be a single point if $z+\varepsilon=f^{q_{\varepsilon}-q_{n}}(z)$; we will assume this is not the case in what follows.

Since $f^{q_{\varepsilon}}$ takes $K_{\varepsilon}$ to the interval of the same length $\left[f^{q_{\varepsilon}}(z), z+\varepsilon\right] \subset \mathfrak{I}_{\varepsilon} \backslash f^{q_{n}}\left(W_{\varepsilon}\right)$, we have $U(x)=f^{q_{\varepsilon}}(x)$ if $x \in K_{\varepsilon}$. Now $f^{q_{\varepsilon}+q_{n}}$ takes the interior of $V_{\varepsilon}$ to the open

\footnotetext{
${ }^{1}$ For the study of combinatorial, spectral and ergodic properties of three-interval exchange transformations and general background on interval exchange maps see [FHZ1, FHZ2, FHZ3, FHZ4 [FHZ5] and references therein.
} 
interval of the same length $\left(f^{q_{n}}(z+\varepsilon), f^{q_{\varepsilon}}(z)\right) \subset \mathfrak{I}_{\varepsilon}$. Therefore, we obtain the following expression for the first return map $U$ of $\mathfrak{I}_{\mathcal{E}}$ :

$$
U(x)= \begin{cases}f^{q_{\varepsilon}}(x) & \text { if } x \in K_{\varepsilon} \\ f^{q_{\varepsilon}+q_{n}}(x) & \text { if } x \in \operatorname{int}\left(V_{\varepsilon}\right) ; \\ f^{q_{n}}(x) & \text { if } x \in W_{\varepsilon} .\end{cases}
$$

The collection of intervals

$$
\left\{K_{\varepsilon}, f\left(K_{\varepsilon}\right), \cdots, f^{q_{\varepsilon}-1}\left(K_{\varepsilon}\right), V_{\varepsilon}, f\left(V_{\varepsilon}\right), \cdots, f^{q_{\varepsilon}+q_{n}-1}\left(V_{\varepsilon}\right), W_{\varepsilon}, f\left(W_{\varepsilon}\right), \cdots, f^{q_{n}-1}\left(W_{\varepsilon}\right)\right\}
$$

covers the circle and the intersection of any two of these intervals is either empty or consists of a single point. This fact implies that for $0<k \leq q_{n}$ we have

$$
\mu\left\{\omega \in S^{1}: \tau_{\varepsilon}(\omega)=k\right\}=\left|K_{\varepsilon}\right|+\left|V_{\varepsilon}\right|+\left|W_{\varepsilon}\right|=\left|\mathfrak{I}_{\varepsilon}\right|=\varepsilon,
$$

for $q_{n}<k \leq q_{\varepsilon}$,

$$
\mu\left\{\omega \in S^{1}: \tau_{\varepsilon}(\omega)=k\right\}=\left|K_{\varepsilon}\right|+\left|V_{\varepsilon}\right|=\left|T^{-1}\left(I_{n}\right)\right|=\left|I_{n}\right|,
$$

and for $q_{\varepsilon}<k \leq q_{\varepsilon}+q_{n}$,

$$
\mu\left\{\omega \in S^{1}: \tau_{\varepsilon}(\omega)=k\right\}=\left|V_{\varepsilon}\right| .
$$

These observations readily give

Proposition 8. The distribution function of $X_{\varepsilon}$ is given by

$$
\begin{aligned}
& \text { (8) } \quad F_{\varepsilon}(t)=\left\{\begin{array}{lr}
k \varepsilon & \text { if } k \varepsilon \leq t<(k+1) \varepsilon, \\
& \text { and } 0 \leq k<q_{n} ; \\
\left(k-q_{n}\right)\left|I_{n}\right|+q_{n} \varepsilon & \text { if } k \varepsilon \leq t<(k+1) \varepsilon, \\
& \text { and } q_{n} \leq k<q_{\varepsilon} ; \\
\left(k-q_{\varepsilon}\right)\left|V_{\varepsilon}\right|+\left(q_{\varepsilon}-q_{n}\right)\left|I_{n}\right|+q_{n} \varepsilon & \text { if } k \varepsilon \leq t<(k+1) \varepsilon, \\
\text { and } q_{\varepsilon} \leq k<q_{\varepsilon}+q_{n} ;
\end{array}\right. \\
& F_{\varepsilon}(t)=0 \text { if } t \leq 0, \text { and } F_{\varepsilon}(t)=q_{n}\left|V_{\varepsilon}\right|+\left(q_{\varepsilon}-q_{n}\right)\left|I_{n}\right|+q_{n} \varepsilon=1 \text { if } t \geq\left(q_{\varepsilon}+q_{n}\right) \varepsilon .
\end{aligned}
$$

In order to study pointwise convergence of $F_{\varepsilon}(t)$ as $\varepsilon \rightarrow 0$, we approximate $F_{\varepsilon}$ by the continuous piecewise linear function given by

$$
L_{\varepsilon}(t)= \begin{cases}t & \text { if } 0 \leq t<q_{n} \varepsilon \\ \left(t-q_{n} \varepsilon\right) \frac{\left|I_{n}\right|}{\varepsilon}+q_{n} \varepsilon & \text { if } q_{n} \varepsilon \leq t<q_{\varepsilon} \varepsilon \\ \left(t-q_{\varepsilon} \varepsilon\right) \frac{\left|V_{\varepsilon}\right|}{\varepsilon}+\left(q_{\varepsilon}-q_{n}\right)\left|I_{n}\right|+q_{n} \varepsilon & \text { if } q_{\varepsilon} \varepsilon \leq t<\left(q_{\varepsilon}+q_{n}\right) \varepsilon ;\end{cases}
$$

$L_{\varepsilon}(t)=0$ for $t \leq 0$ and $L_{\varepsilon}(t)=1$ for $t \geq\left(q_{\varepsilon}+q_{n}\right) \varepsilon$. Note that $0 \leq L_{\varepsilon}(t)-F_{\varepsilon}(t) \leq \varepsilon$ for all $t \in \mathbb{R}$. Therefore, $F_{\varepsilon}$ will converge pointwise or uniformly on compact sets if and only if $L_{\varepsilon}$ does.

\section{The CASE $\Gamma^{n_{k}-1}(\alpha, \cdot) \rightarrow(\theta, \nu)$ FOR SOME $\theta>0$ AND $\nu<1$, AND THE PROOF OF THEOREM 3}

Let $\Sigma$ denote a subsequence $\left\{n_{k}\right\}$ of $n \in \mathbb{N}$ and suppose the rotation number $\alpha$ satisfies

$$
\lim _{n \rightarrow \infty, n \in \Sigma} \Gamma^{n-1}(\alpha, \cdot)=(\theta, \nu)
$$


for some $\theta>0$ and $\nu<1$. We will take a particular subsequence of $\varepsilon$ given by $\varepsilon_{n}=c\left|I_{n-1}\right|$ with $\theta<c<1$ for $n \in \Sigma$. Let $N>0$ be given such that $\left|I_{n}\right|<\varepsilon_{n}<\left|I_{n-1}\right|$ for all $n \in \Sigma$ larger than $N$. Fix $n>N$ in what follows.

Inducing $f$ on $\mathfrak{I}_{\varepsilon}$ as in the previous section we note that $a_{\varepsilon_{n}}$ is the maximum number of copies of $I_{n}$ that one can fit in $I_{n-1} \backslash \mathfrak{I}_{\varepsilon_{n}}$ plus 1 . Therefore $a_{\varepsilon_{n}}=$ $1+\left[\left(\left|I_{n-1}\right|-\varepsilon_{n}\right) /\left|I_{n}\right|\right]$, where [.] denotes the integer part. Increasing $N$ if necessary, we obtain

$$
a_{\varepsilon_{n}}=1+\left[\frac{1-c}{\theta}\right]=a_{c, \theta}
$$

which does not depend on $n \in \Sigma$ for $n>N$. Define $V_{n}=V_{\varepsilon_{n}}$ and $L_{n}(t)=L_{\varepsilon_{n}}(t)$. From the definition of $L_{\varepsilon}$ in (9) we obtain

$$
L_{n}(t)= \begin{cases}t & \text { if } 0 \leq t<c q_{n}\left|I_{n-1}\right| ; \\ \left(t-c q_{n}\left|I_{n-1}\right|\right) \frac{\left|I_{n}\right|}{c\left|I_{n-1}\right|}+c q_{n}\left|I_{n-1}\right| & \text { if } c q_{n}\left|I_{n-1}\right| \leq t \text { and } \\ & t<c\left(a_{c, \theta} q_{n}+q_{n-1}\right)\left|I_{n-1}\right| ; \\ \left(t-c\left(a_{c, \theta} q_{n}+q_{n-1}\right)\left|I_{n-1}\right|\right) \frac{\left|V_{n}\right|}{c\left|I_{n-1}\right|} & \text { if } c\left(a_{c, \theta} q_{n}+q_{n-1}\right)\left|I_{n-1}\right| \\ +\left(\left(a_{c, \theta}-1\right) q_{n}+q_{n-1}\right)\left|I_{n}\right| & \leq t \text { and } t< \\ +c q_{n}\left|I_{n-1}\right| & c\left(\left(1+a_{c, \theta}\right) q_{n}+q_{n-1}\right)\left|I_{n-1}\right| ;\end{cases}
$$

$L_{n}(t)=0$ for $t \leq 0$ and $L_{n}(t)=1$ for $t \geq c\left(\left(1+a_{c, \theta}\right) q_{n}+q_{n-1}\right)\left|I_{n-1}\right|$.

From the computation of the induced map $U$ on $\mathfrak{I}_{\mathcal{E}_{n}}$ and the definition of $a_{c, \theta}$ we obtain

$$
\left|V_{n}\right|=\left|\left(I_{n-1} \backslash \mathfrak{I}_{\mathcal{\varepsilon}_{n}}\right) \backslash\left(\bigcup_{k=1}^{a_{c, \theta}-1} T^{k}\left(I_{n}\right)\right)\right|=\left|I_{n-1}\right|-\varepsilon_{n}-\left(a_{c, \theta}-1\right)\left|I_{n}\right|
$$

if $a_{c, \theta}>1$, and $\left|V_{n}\right|=\left|I_{n-1} \backslash \mathfrak{I}_{\varepsilon_{n}}\right|=\left|I_{n-1}\right|-\varepsilon_{n}$ if $a_{c, \theta}=1$. Since for $n \in \Sigma$, $\left|I_{n}\right| /\left|I_{n-1}\right|$ is asymptotically equal to $\theta$, we have

$$
\lim _{n \rightarrow \infty, n \in \Sigma} \frac{\left|V_{n}\right|}{\left|I_{n-1}\right|}=(1-c)-\left(a_{c, \theta}-1\right) \theta=(1-c)-\theta\left[\frac{1-c}{\theta}\right]=\theta\left\{\frac{1-c}{\theta}\right\}
$$

where $\{\cdot\}$ denotes fractional part. From (6) and the identity $q_{n}\left|I_{n-1}\right|+q_{n-1}\left|I_{n}\right|=1$ we have

$$
\lim _{n \rightarrow \infty, n \in \Sigma} q_{n}\left|I_{n-1}\right|=\frac{1}{1+\theta \nu} \quad \text { and } \quad \lim _{n \rightarrow \infty, n \in \Sigma} q_{n-1}\left|I_{n-1}\right|=\frac{\nu}{1+\theta \nu} .
$$

Define the constants

$$
d_{1}=\frac{c}{1+\theta \nu}, \quad d_{2}=\left(a_{c, \theta}+\nu\right) d_{1} \quad \text { and } \quad d_{3}=d_{1}+d_{2} .
$$

From the expression of $L_{n}(t)$ in (10) and (12) we obtain the next result.

Theorem 9. Let $\Sigma$ be a subsequence of $n \in \mathbb{N}$. Suppose the rotation number $\alpha$ satisfies $\lim _{n \rightarrow \infty, n \in \Sigma} \Gamma^{n-1}(\alpha, \cdot)=(\theta, \nu)$, for some $\theta>0$ and $\nu<1$. Let $\varepsilon_{n}=$ $c\left|I_{n-1}\right|$ with $\theta<c<1$ for $n \in \Sigma$. The distribution functions $F_{\varepsilon_{n}}(t)$ of $X_{\varepsilon_{n}}(\cdot)=$ 
$\varepsilon_{n} \tau_{\varepsilon_{n}}(\cdot)$ converge uniformly to the continuous piecewise linear function

$$
L(t)= \begin{cases}t & \text { if } 0 \leq t<d_{1} ; \\ \left(t-d_{1}\right) \frac{\theta}{c}+d_{1} & \text { if } d_{1} \leq t<d_{2} ; \\ \left(t-d_{2}\right) \frac{\theta}{c}\left\{\frac{1-c}{\theta}\right\}+\left(d_{2}-d_{1}\right) \frac{\theta}{c}+d_{1} & \text { if } d_{2} \leq t<d_{3} ;\end{cases}
$$

$L(t)=0$ for $t<0$ and $L(t)=1$ for $t \geq d_{3}$, where the constants $d_{1}, d_{2}, d_{3}$ are given by (13); see Figure 2

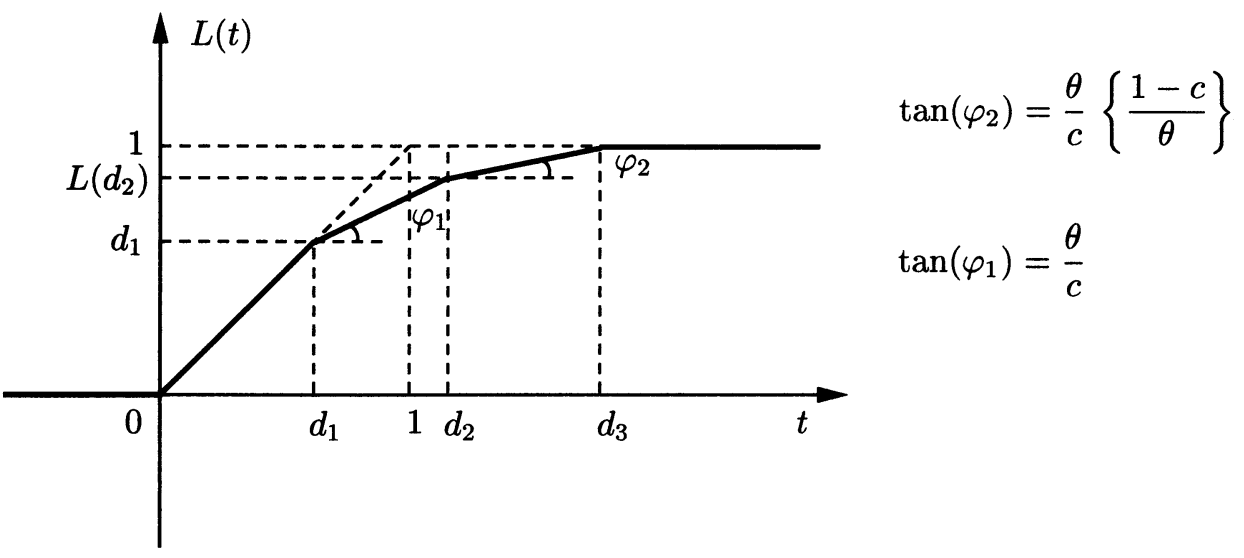

Figure 2. The graph of $L(t)$.

By taking subsequences $\varepsilon_{n}=\left|I_{n-1}\right|$ and $\varepsilon_{n}^{\prime}=c\left|I_{n-1}\right|$, with $(-1+\sqrt{5}) / 2<c<1$, Theorem 9 together with Lemma 7 gives

Lemma 10. If $X_{\varepsilon}$ converges in law when $\varepsilon \rightarrow 0$ then the rotation number $\alpha$ satisfies necessarily $\lim _{n \rightarrow \infty} G^{n}(\alpha)=0$.

Now we discuss the problem of tightness of the distributions of $X_{\varepsilon}$ for arbitrary $\varepsilon>0$, when the rotation number $\alpha$ is of bounded type.

Proof of Theorem 3 . Using Theorem 2 we need only to show that if $\alpha$ is of bounded type then the distributions of $X_{\varepsilon}$ are tight. Suppose $a_{n} \leq a$ for all $n>0$. Then $a_{\varepsilon} \leq$ $a$ for all $\varepsilon>0$. The support of the distribution of $X_{\varepsilon}$ is contained in $\left[0,\left(q_{\varepsilon}+q_{n}\right) \varepsilon\right]$. However, since $q_{n-1}\left|I_{n}\right|+q_{n}\left|I_{n-1}\right|=1$ for all $n>0$, we have

$$
\left(q_{\varepsilon}+q_{n}\right) \varepsilon \leq\left((a+1) q_{n}+q_{n-1}\right)\left|I_{n-1}\right| \leq a+2 .
$$

Therefore the distributions of $X_{\varepsilon}$ for $\varepsilon>0$ have uniformly bounded support in this case.

4. The CASE $G^{n_{k}-1}(\alpha) \rightarrow 0$ And the Proof of Theorems 1$]$ and 2

Let $\Sigma$ be a subsequence of $n \in \mathbb{N}$ such that the rotation number $\alpha$ satisfies

$$
\lim _{n \rightarrow \infty, n \in \Sigma} G^{n-1}(\alpha)=0 .
$$


Take the subsequence of $\varepsilon$ given by $\varepsilon_{n}=c\left|I_{n-1}\right|$ with $0<c<1$ for $n \in \Sigma$. Let $N>0$ be given such that $\left|I_{n}\right|<\varepsilon_{n}<\left|I_{n-1}\right|$ for all $n \in \Sigma$ larger than $N$. Fix $n>N$ in what follows. As in the previous section we have

$$
a_{\varepsilon_{n}}=1+\left[\frac{\left|I_{n-1}\right|-\varepsilon_{n}}{\left|I_{n}\right|}\right]=1+\left[\frac{(1-c)\left|I_{n-1}\right|}{\left|I_{n}\right|}\right],
$$

where [.] denotes the integer part. Define $V_{n}=V_{\varepsilon_{n}}$ and $L_{n}(t)=L_{\varepsilon_{n}}(t)$, where $L_{\varepsilon}$ in given by (9). Therefore we get

$$
L_{n}(t)= \begin{cases}t & \text { if } 0 \leq t<c q_{n}\left|I_{n-1}\right| \\ \left(t-c q_{n}\left|I_{n-1}\right|\right) \frac{\left|I_{n}\right|}{c\left|I_{n-1}\right|} & \text { if } c q_{n}\left|I_{n-1}\right| \leq t<c q_{\varepsilon_{n}}\left|I_{n-1}\right| \\ +c q_{n}\left|I_{n-1}\right| & \\ \left(t-c q_{\varepsilon_{n}}\left|I_{n-1}\right|\right) \frac{\left|V_{n}\right|}{c\left|I_{n-1}\right|} & \text { if } c q_{\varepsilon_{n}}\left|I_{n-1}\right| \leq t \\ +\left(q_{\varepsilon_{n}}-q_{n}\right)\left|I_{n}\right|+c q_{n}\left|I_{n-1}\right| & <c\left(q_{\varepsilon_{n}}+q_{n}\right)\left|I_{n-1}\right|\end{cases}
$$

$L_{n}(t)=0$ for $t \leq 0$ and $L_{n}(t)=1$ for $t \geq c\left(q_{\varepsilon_{n}}+q_{n-1}\right)\left|I_{n-1}\right|$, where $q_{\varepsilon_{n}}=$ $a_{\varepsilon_{n}} q_{n}+q_{n-1}$.

Proof of Theorem 2. Since $q_{n-1}\left|I_{n-1}\right|$ is bounded, $\lim _{n \rightarrow \infty, n \in \Sigma} q_{n}\left|I_{n-1}\right|=1$, and $a_{\varepsilon_{n}}$ diverges to infinity, we conclude that

$$
\lim _{n \rightarrow \infty, n \in \Sigma} q_{\varepsilon_{n}}\left|I_{n-1}\right|=\infty .
$$

Since $\left|V_{n}\right| /\left|I_{n-1}\right|<\left|I_{n}\right| /\left|I_{n-1}\right|$ convergent to zero, from the expression (15) we see that $L_{n}(t)$ converges uniformly on compact sets to $F_{\mu}(t)$ of (1). Hence the same holds for $F_{\varepsilon_{n}}$.

Proof of Theorem 1. By Lemma 10 we need only to worry about the case when the rotation number $\alpha$ satisfies $\lim _{n \rightarrow \infty} G^{n}(\alpha)=0$. However, by taking subsequences $\varepsilon_{n}=\left|I_{n-1}\right|$ and $\varepsilon_{n}^{\prime}=c\left|I_{n-1}\right|$, with $0<c<1$, on one hand we have $F_{\varepsilon_{n}}$ converges uniformly to the uniform distribution on $[0,1]$ (see Section 1), whereas by the proof of Theorem $2 F_{\varepsilon_{n}^{\prime}}$ converges uniformly on compact sets to the function $F_{\mu}(t)$ of (1). Therefore there cannot be convergence in law of $X_{\varepsilon}$ as $\varepsilon \rightarrow 0$.

\section{Another time scale in the Case $G^{n_{k}-1}(\alpha) \rightarrow 0$ AND THE PROOF OF THEOREM 4}

Here we show that in the case $\lim _{n \rightarrow \infty, n \in \Sigma} G^{n-1}(\alpha)=0$, for $\Sigma$ a subsequence of $n \in \mathbb{N}$, another scale $\gamma_{\varepsilon} \neq \mu\left(\mathfrak{I}_{\varepsilon}\right)$ can be used to obtain convergence in law of the corresponding rescaled hitting time distribution for certain subsequences $\varepsilon_{n}$ of $\varepsilon \rightarrow 0$.

Consider the random variable $Y_{\varepsilon}(\cdot)=\gamma_{\varepsilon} \tau_{\varepsilon}(\cdot)$. Let $F_{\varepsilon}^{Y}(t)$ denote the distribution function of $Y_{\varepsilon}$. Then we note that $F_{\varepsilon}^{Y}(t)=F_{\varepsilon}\left(\varepsilon t / \gamma_{\varepsilon}\right)$, where $F_{\varepsilon}(t)$ is given by (8). (We remark that we are still assuming as in Section 1 that $f$ is the rigid rotation by $\alpha$ and hence $\mu\left(\mathfrak{I}_{\varepsilon}\right)=\varepsilon$.) By Proposition 8 we may approximate $F_{\varepsilon}^{Y}(t)$ by the continuous piecewise linear function $L_{\varepsilon}^{Y}(t)=L_{\varepsilon}\left(\varepsilon t / \gamma_{\varepsilon}\right)$, where $L_{\varepsilon}(t)$ is given by (9). For $0<c<1$, define $\varepsilon_{n}=c\left|I_{n-1}\right|$ and take $\gamma_{\varepsilon_{n}}=\left|I_{n}\right|$. Define as before $V_{n}=V_{\varepsilon_{n}}$ 
and $q_{\varepsilon_{n}}=a_{\varepsilon_{n}} q_{n}+q_{n-1}$. Then $L_{n}^{Y}(t)=L_{\varepsilon_{n}}^{Y}(t)$ is given by

$$
L_{n}^{Y}(t)= \begin{cases}c \frac{\left|I_{n-1}\right|}{\left|I_{n}\right|} t & \text { if } 0 \leq t<q_{n}\left|I_{n}\right| \\ t-q_{n}\left|I_{n}\right|+c q_{n}\left|I_{n-1}\right| & \text { if } q_{n}\left|I_{n}\right| \leq t<q_{\varepsilon_{n}}\left|I_{n}\right| \\ \left(t-q_{\varepsilon_{n}}\left|I_{n}\right|\right) \frac{\left|V_{n}\right|}{\left|I_{n}\right|} & \text { if } q_{\varepsilon_{n}}\left|I_{n}\right| \leq t<\left(q_{\varepsilon_{n}}+q_{n}\right)\left|I_{n}\right| \\ +\left(q_{\varepsilon_{n}}-q_{n}\right)\left|I_{n}\right|+c q_{n}\left|I_{n-1}\right| & \end{cases}
$$

$L_{n}^{Y}(t)=0$ for $t \leq 0$ and $L_{n}^{Y}(t)=1$ for $t \geq\left(q_{\varepsilon_{n}}+q_{n}\right)\left|I_{n}\right|$.

Proof of Theorem 4 . We recall that

$$
\lim _{n \rightarrow \infty, n \in \Sigma} q_{n}\left|I_{n}\right|=0 \quad \text { and } \quad \lim _{n \rightarrow \infty, n \in \Sigma} q_{n}\left|I_{n-1}\right|=1 .
$$

Since for $n \in \Sigma$ we have

$$
a_{\varepsilon_{n}} \frac{\left|I_{n}\right|}{\left|I_{n-1}\right|}=\left(1+\left[\frac{(1-c)\left|I_{n-1}\right|}{\left|I_{n}\right|}\right]\right) \frac{\left|I_{n}\right|}{\left|I_{n-1}\right|} \rightarrow 1-c
$$

we see that

$$
q_{\varepsilon_{n}}\left|I_{n}\right|=\left(a_{\varepsilon_{n}} q_{n}+q_{n-1}\right)\left|I_{n}\right| \rightarrow 1-c .
$$

Since $\left|V_{n}\right| /\left|I_{n}\right|$ is bounded above by 1 and $\left(q_{\varepsilon_{n}}+q_{n}\right)\left|I_{n}\right|$ also converges to $1-c$, we conclude from (16) that $L_{n}^{Y}(t)$ converges pointwise to the limit function $F_{\gamma}(t)$ of (2).

\section{The hitting time Point Process}

Define the hitting time point process $\mathcal{T}_{\varepsilon}$ of $\mathfrak{I}_{\varepsilon}$ with time scale $\gamma_{\varepsilon}$ to be the map $\mathcal{T}_{\varepsilon}: S^{1} \rightarrow \mathcal{M}[0, \infty)$ given by

$$
\mathcal{T}_{\varepsilon}(\omega)=\sum_{k>0} \chi_{\mathfrak{I}_{\varepsilon}}\left(f^{k} \omega\right) \delta_{k \cdot \gamma_{\varepsilon}}
$$

where $\delta_{t}$ denotes the Dirac measure at the point $t>0, \mathcal{M}[0, \infty)$ denotes the $\sigma$ finite Borel measures on $[0, \infty)$, and $\chi_{\mathfrak{I}_{\varepsilon}}$ denotes the indicator function of $\mathfrak{I}_{\varepsilon}$. The support of $\mathcal{T}_{\varepsilon}$ defines the sequence of rescaled hitting times

$$
X_{\varepsilon}^{(1)}(\omega)=\gamma_{\varepsilon} \tau_{\varepsilon}(\omega), X_{\varepsilon}^{(2)}(\omega)=\gamma_{\varepsilon} \tau_{\varepsilon}^{(2)}(\omega), \ldots
$$

In $\mathrm{CF}$ ] we studied convergence in law of $\mathcal{T}_{\varepsilon}$ as $\varepsilon \rightarrow 0$ in the particular case of $\varepsilon=\varepsilon_{n}=\left|I_{n}\right|+\left|I_{n-1}\right|$ and $\gamma_{\varepsilon}=\varepsilon_{n}$. For this particular choice of $\varepsilon_{n}$ we obtained

Proposition 11. The point process $\mathcal{T}_{\varepsilon_{n_{k}}}$ converges in law if and only if either

(a) $\lim _{k \rightarrow \infty} G^{n_{k}-1}(\alpha)=0$; or

(b) there exist $\theta>0$ and $\nu<1$ such that $\lim _{k \rightarrow \infty} \Gamma^{n_{k}-1}(\alpha, \cdot) \rightarrow(\theta, \nu)$.

Here we address the problem of choosing different subsequences $\varepsilon_{n}$ and different scales $\gamma_{\varepsilon_{n}}$. Let $D_{\varepsilon}^{(1)}=X_{\varepsilon}^{(1)}$, and for $k>1, D_{\varepsilon}^{(k)}=X_{\varepsilon}^{(k)}-X_{\varepsilon}^{(k-1)}$. In order to study convergence in law of (17) we need to study convergence of the finite-dimensional joint distributions of $\left\{D_{\varepsilon}^{(k)}\right\}$. For $0<k_{1}<\cdots<k_{r}$ define

$$
F_{\varepsilon}^{\left(k_{1}, \cdots, k_{r}\right)}\left(t_{1}, \cdots, t_{r}\right)=\mu\left\{\omega \in S^{1}: D_{\varepsilon}^{\left(k_{s}\right)}(\omega) \leq t_{s}, s=1, \cdots, r\right\} .
$$

We start with the case of $F_{\varepsilon}^{(k)}(t)$. For $k=1$, we have $F_{\varepsilon}^{(1)}(t)=F_{\varepsilon}\left(\varepsilon t / \gamma_{\varepsilon}\right)$, where $F_{\varepsilon}(t)$ is given by (8) . (Here again we may assume $f$ is the rigid rotation by $\alpha$ and 
then $\mu\left(\mathfrak{I}_{\varepsilon}\right)=\varepsilon$.) Consider the continuous piecewise linear approximation of $F_{\varepsilon}^{(1)}(t)$ given by $L_{\varepsilon}^{(1)}(t)=L_{\varepsilon}\left(\varepsilon t / \gamma_{\varepsilon}\right)$, where $L_{\varepsilon}(t)$ is defined in (9).

In order to have pointwise convergence of $L_{\varepsilon_{n}}^{(1)}(t)$, a necessary condition is that either $\left|I_{n^{\prime}-1}\right| / \gamma_{\varepsilon_{n}}$ converges (where $n^{\prime}$ is the unique solution of $\left|I_{n^{\prime}}\right|<\varepsilon_{n} \leq\left|I_{n^{\prime}-1}\right|$ ), or $\left|I_{n^{\prime}}\right| / \gamma_{\varepsilon_{n}}$ converges. Therefore the interesting cases to consider are $\varepsilon_{n}=c\left|I_{n-1}\right|$ with $0<c \leq 1$, where $\gamma_{\varepsilon_{n}}$ satisfies either (i) $\gamma_{\varepsilon_{n}}=\varepsilon_{n}$, or (ii) $\gamma_{\varepsilon_{n}}=\left|I_{n}\right|$; and $n$ belongs to an infinite subset $\Sigma$ of $\mathbb{N}$.

The study of convergence of $L_{\varepsilon_{n}}^{(1)}(t)$ in the above cases have been carried out in the previous sections, and again pointwise convergence occurs only in the cases (a) and (b) of Proposition 11. Since the case $c=1$ is very similar to what is done in [CF], we will fix $0<c<1$. We start by choosing $\gamma_{\varepsilon_{n}}=\varepsilon_{n}$.

Case (b) of Proposition [1]. Let $\Sigma$ be a subsequence of $n \in \mathbb{N}$ such that

$$
\lim _{n \rightarrow \infty, n \in \Sigma} \Gamma^{n-1}(\alpha, \cdot)=(\theta, \nu),
$$

for some $\theta>0$ and $\nu<1$. Note that we must have $c>\theta$. On $\mathfrak{I}_{\varepsilon_{n}}$ there are only three return times $q_{n}, q_{\varepsilon_{n}}$ and $q_{\varepsilon_{n}}+q_{n}$, assumed respectively on $W_{n}=W_{\varepsilon_{n}}$, $K_{n}=K_{\varepsilon_{n}}$ and $V_{n}=V_{\varepsilon_{n}}$. Therefore, for $k>1, D_{\varepsilon_{n}}^{(k)}$ assumes only the values $q_{n} \varepsilon_{n}$, $q_{\varepsilon_{n}} \varepsilon_{n}$ and $\left(q_{\varepsilon_{n}}+q_{n}\right) \varepsilon_{n}$. Rescaling the interval $\mathfrak{I}_{\varepsilon_{n}}$ to $[0,1]$ using an affine map, we see that the return map $U$ of $\mathfrak{I}_{\varepsilon_{n}}$ becomes an interval exchange map $\widetilde{U}_{n}$ of $[0,1]$ with parameters $\left(\left|K_{n}\right| / \varepsilon_{n},\left|I_{n}\right| / \varepsilon_{n}\right)$ and permutation (321). By (11) and the fact that $\left|K_{n}\right|+\left|V_{n}\right|=\left|I_{n}\right|$ we get

$$
\begin{gathered}
\frac{\left|K_{n}\right|}{\left|\varepsilon_{n}\right|} \rightarrow \lambda_{1}=\frac{\theta}{c}\left(1-\left\{\frac{1-c}{\theta}\right\}\right), \quad \frac{\left|V_{n}\right|}{\left|\varepsilon_{n}\right|} \rightarrow \lambda_{2}=\frac{\theta}{c}\left\{\frac{1-c}{\theta}\right\}, \\
\text { and } \frac{\left|W_{n}\right|}{\left|\varepsilon_{n}\right|} \rightarrow \lambda_{3}=1-\lambda_{1}-\lambda_{2}=1-\frac{\theta}{c}
\end{gathered}
$$

Therefore we see that $\widetilde{U}_{n}$ converges to the interval exchange map $U_{c, \theta}$ of parameters $\left(\lambda_{1}, \lambda_{1}+\lambda_{2}\right)$ and permutation (321), when $(1-c)$ is not divisible by $\theta$. When $(1-c)$ is divisible by $\theta, \widetilde{U}_{n}$ converges to the rigid rotation of the circle of rotation number $(c-\theta) / c$, which we will also denote by $U_{c, \theta}$.

Restricted to $\mathfrak{I}_{\varepsilon_{n}}$ we have $\left\{\omega \in \mathfrak{I}_{\varepsilon_{n}}: D_{\varepsilon_{n}}^{(k)}(\omega)=q_{n} \varepsilon_{n}\right\}=U^{-k+1}\left(W_{n}\right)$ and $\left\{\omega \in \mathfrak{I}_{\varepsilon_{n}}: D_{\varepsilon_{n}}^{(k)}(\omega)=q_{\varepsilon_{n}} \varepsilon_{n}\right\}=U^{-k+1}\left(K_{n}\right)$. Building the Rokhlin-Kakutani tower of $f$ on top of $\mathfrak{I}_{\varepsilon_{n}}$ we conclude that

$$
\begin{aligned}
\mu\{\omega \in & \left.S^{1}: D_{\varepsilon_{n}}^{(k)}(\omega)=q_{n} \varepsilon_{n}\right\} \\
= & q_{n} \mu\left(U^{-k+1}\left(W_{n}\right)\right)+\left(q_{\varepsilon_{n}}-q_{n}\right) \mu\left(U^{-k+1}\left(W_{n}\right) \cap T^{-1}\left(I_{n}\right)\right) \\
& +q_{n} \mu\left(U^{-k+1}\left(W_{n}\right) \cap V_{n}\right) .
\end{aligned}
$$

Similarly we have

$$
\begin{aligned}
\mu\{\omega \in & \left.S^{1}: D_{\varepsilon_{n}}^{(k)}(\omega)=q_{\varepsilon_{n}} \varepsilon_{n}\right\} \\
= & q_{n} \mu\left(U^{-k+1}\left(K_{n}\right)\right)+\left(q_{\varepsilon_{n}}-q_{n}\right) \mu\left(U^{-k+1}\left(K_{n}\right) \cap T^{-1}\left(I_{n}\right)\right) \\
& +q_{n} \mu\left(U^{-k+1}\left(K_{n}\right) \cap V_{n}\right) .
\end{aligned}
$$

Multiplying and dividing by $\varepsilon_{n}$ on the right hand side of both (18) and (19), and using the fact that $q_{n} \varepsilon_{n} \rightarrow d_{1}$ and $q_{\varepsilon_{n}} \varepsilon_{n} \rightarrow d_{2}$, where $d_{1}, d_{2}$ are given by (13) we 
obtain

(20)

$$
\begin{aligned}
\mu\left\{\omega \in S^{1}: D_{\varepsilon_{n}}^{(k)}(\omega)=q_{n} \varepsilon_{n}\right\} \rightarrow \eta_{k, 1}=d_{1} & \lambda_{3}+\left(d_{2}-d_{1}\right) \mu\left(U_{c, \theta}^{-k+1}(W) \cap I\right) \\
& +d_{1} \mu\left(U_{c, \theta}^{-k+1}(W) \cap V\right)
\end{aligned}
$$

where we define $K=\left[0, \lambda_{1}\right], V=\left[\lambda_{1}, \lambda_{1}+\lambda_{2}\right], W=\left[\lambda_{1}+\lambda_{2}, 1\right]$ and $I=K \cup V=$ $\left[0, \lambda_{1}+\lambda_{2}\right]$. Similarly,

$$
\begin{gathered}
\mu\left\{\omega \in S^{1}: D_{\varepsilon_{n}}^{(k)}(\omega)=q_{\varepsilon_{n}} \varepsilon_{n}\right\} \rightarrow \eta_{k, 2}=d_{1} \lambda_{1}+\left(d_{2}-d_{1}\right) \mu\left(U_{c, \theta}^{-k+1}(K) \cap I\right) \\
+d_{1} \mu\left(U_{c, \theta}^{-k+1}(K) \cap V\right) .
\end{gathered}
$$

The above shows that $F_{\varepsilon_{n}}^{(k)}(t)$ converges in law for every $k>0$. Figure 3 shows the graph of $F^{(k)}(t)=\lim _{n \rightarrow \infty} F_{\varepsilon_{n}}^{(k)}(t)$, for $k>1$, and Figure 2 shows the graph of $L(t)=\lim _{n \rightarrow \infty} F_{\varepsilon_{n}}^{(1)}(t)$.

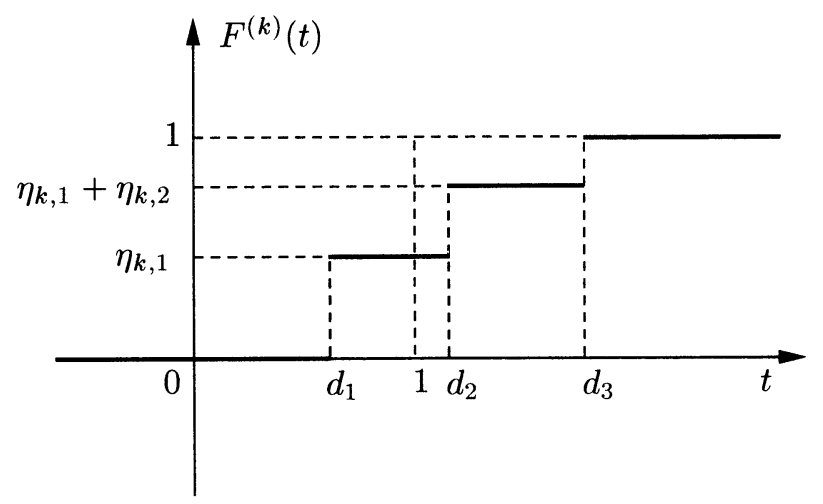

Figure 3. The graph of $F^{(k)}(t)$, for $k>1$.

Now we consider the case of $F_{\varepsilon_{n}}^{\left(k_{1}, \cdots, k_{r}\right)}\left(t_{1}, \cdots, t_{r}\right)$, where $1<k_{1}<\cdots<k_{r}$. Introducing the indices $\ell_{n, 1}=q_{n} \varepsilon_{n}$ and $\ell_{n, 2}=q_{\varepsilon_{n}} \varepsilon_{n}$ we have $\ell_{n, i} \rightarrow d_{i}$ for $i=1,2$. Define $Z_{n, 1}=W_{n}$ and $Z_{n, 2}=K_{n}$, and also $Z_{1}=W$ and $Z_{2}=K$. First we note that restricted to $\mathfrak{I}_{\mathcal{E}_{n}}$ we have

$$
\left\{\omega \in \mathfrak{I}_{\varepsilon_{n}}: D_{\varepsilon_{n}}^{\left(k_{s}\right)}(\omega)=\ell_{n, i_{s}}, s=1, \cdots, r\right\}=\bigcap_{s=1}^{r} U^{-k_{s}+1}\left(Z_{n, i_{s}}\right),
$$

for any choice of $i_{s} \in\{1,2\}, s=1, \cdots, r$. From the Rokhlin-Kakutani tower on top of $\mathfrak{I}_{\varepsilon_{n}}$ we conclude that

$$
\begin{aligned}
\mu\{\omega \in & \left.S^{1}: D_{\varepsilon_{n}}^{\left(k_{s}\right)}(\omega)=\ell_{n, i_{s}}, s=1, \cdots, r\right\} \\
= & q_{n} \mu\left(\bigcap_{s=1}^{r} U^{-k_{s}+1}\left(Z_{n, i_{s}}\right)\right)+\left(q_{\varepsilon_{n}}-q_{n}\right) \mu\left(\bigcap_{s=1}^{r} U^{-k_{s}+1}\left(Z_{n, i_{s}}\right) \cap T^{-1}\left(I_{n}\right)\right) \\
& +q_{n} \mu\left(\bigcap_{s=1}^{r} U^{-k_{s}+1}\left(Z_{n, i_{s}}\right) \cap V_{n}\right) .
\end{aligned}
$$


Therefore we obtain

$$
\begin{aligned}
\mu\left\{\omega \in S^{1}:\right. & \left.D_{\varepsilon_{n}}^{\left(k_{s}\right)}(\omega)=\ell_{n, i_{s}}, s=1, \cdots, r\right\} \rightarrow d_{1} \mu\left(\bigcap_{s=1}^{r} U_{c, \theta}^{-k_{s}+1}\left(Z_{i_{s}}\right)\right) \\
& +\left(d_{2}-d_{1}\right) \mu\left(\bigcap_{s=1}^{r} U_{c, \theta}^{-k_{s}+1}\left(Z_{i_{s}}\right) \cap I\right)+d_{1} \mu\left(\bigcap_{s=1}^{r} U_{c, \theta}^{-k_{s}+1}\left(Z_{i_{s}}\right) \cap V\right) .
\end{aligned}
$$

This shows that $F_{\varepsilon_{n}}^{\left(k_{1}, \cdots, k_{r}\right)}\left(t_{1}, \cdots, t_{r}\right)$ converges in law for every choice of $1<k_{1}<$ $\cdots<k_{r}$. Finally, for the case of $F_{\varepsilon_{n}}^{\left(1, k_{2}, \cdots, k_{r}\right)}\left(t_{1}, \cdots, t_{r}\right)$ we study the convergence of

(24) $G_{n, i_{2}, \cdots, i_{r}}^{\left(1, k_{2}, \cdots, k_{r}\right)}(t)=\mu\left\{\omega \in S^{1}: D_{\varepsilon_{n}}^{(1)}(\omega) \leq t ; D_{\varepsilon_{n}}^{\left(k_{s}\right)}(\omega)=\ell_{n, i_{s}}, s=2, \cdots, r\right\}$.

Suppose $\varepsilon_{n} k \leq t<\varepsilon_{n}(k+1)$. For $k \leq q_{n}$ we have

$$
G_{n, i_{2}, \cdots, i_{r}}^{\left(1, k_{2}, \cdots, k_{r}\right)}(t)=k \mu\left(\bigcap_{s=2}^{r} U^{-k_{s}+1}\left(Z_{n, i_{s}}\right)\right) .
$$

For $q_{n}<k \leq q_{\varepsilon_{n}}$ we have

$$
\begin{aligned}
G_{n, i_{2}, \cdots, i_{r}}^{\left(1, k_{2}, \cdots, k_{r}\right)}(t)= & q_{n} \mu\left(\bigcap_{s=2}^{r} U^{-k_{s}+1}\left(Z_{n, i_{s}}\right)\right) \\
& +\left(k-q_{n}\right) \mu\left(\bigcap_{s=2}^{r} U^{-k_{s}+1}\left(Z_{n, i_{s}}\right) \cap T^{-1}\left(I_{n}\right)\right) .
\end{aligned}
$$

For $q_{\varepsilon_{n}}<k \leq q_{\varepsilon_{n}}+q_{n}$ we have

$$
\begin{aligned}
G_{n, i_{2}, \cdots, i_{r}}^{\left(1, k_{2}, \cdots, k_{r}\right)}(t)= & q_{n} \mu\left(\bigcap_{s=2}^{r} U^{-k_{s}+1}\left(Z_{n, i_{s}}\right)\right) \\
& +\left(q_{\varepsilon_{n}}-q_{n}\right) \mu\left(\bigcap_{s=2}^{r} U^{-k_{s}+1}\left(Z_{n, i_{s}}\right) \cap T^{-1}\left(I_{n}\right)\right) \\
& +\left(k-q_{\varepsilon_{n}}\right) \mu\left(\bigcap_{s=2}^{r} U^{-k_{s}+1}\left(Z_{n, i_{s}}\right) \cap V_{n}\right) .
\end{aligned}
$$

Therefore we see that $G_{n, i_{2}, \cdots, i_{r}}^{\left(1, k_{2}, \cdots, k_{r}\right)}(t)$ converges uniformly to the continuous piecewise linear function $G_{i_{2}, \cdots, i_{r}}^{\left(1, k_{2}, \cdots, k_{r}\right)}(t)$ given by

$$
\begin{aligned}
& G_{i_{2}, \cdots, i_{r}}^{\left(1, k_{2}, \cdots, k_{r}\right)}(t)= \begin{cases}t \mu(R) & \text { if } \quad 0 \leq t<d_{1} ; \\
\left(t-d_{1}\right) \mu(R \cap I)+d_{1} \mu(R) & \text { if } \quad d_{1} \leq t<d_{2} \\
\left(t-d_{2}\right) \mu(R \cap V) & \text { if } \quad d_{2} \leq t<d_{3} \\
+\left(d_{2}-d_{1}\right) \mu(R \cap I)+d_{1} \mu(R)\end{cases} \\
& G_{i_{2}, \cdots, i_{r}}^{\left(1, k_{2}, \cdots, k_{r}\right)}(t)=0 \quad \text { for } t<0
\end{aligned}
$$

and

$$
G_{i_{2}, \cdots, i_{r}}^{\left(1, k_{2}, \cdots, k_{r}\right)}(t)=d_{1} \mu(R \cap V)+\left(d_{2}-d_{1}\right) \mu(R \cap I)+d_{1} \mu(R) \quad \text { for } t \geq d_{3},
$$

where the constants $d_{1}, d_{2}, d_{3}$ are given by (13) and $R=\bigcap_{s=2}^{r} U_{c, \theta}^{-k_{s}+1}\left(Z_{i_{s}}\right)$. 
The above computations show that the point process $\mathcal{T}_{\varepsilon_{n}}$ with $\gamma_{\varepsilon_{n}}=\varepsilon_{n}$ (under the subsequence $n \in \Sigma$ ) converges in law to a non-stationary, inhomogeneous and non-independent limit point process. For $\gamma_{\varepsilon_{n}}=\left|I_{n}\right|$ the result is the same since $\left|I_{n}\right| / \varepsilon_{n}$ converges to $\theta / c$, and hence the limit point process is just scaled by this factor.

Case (a) of Proposition 11 Let $\Sigma$ be a subsequence of $n \in \mathbb{N}$ such that

$$
\lim _{n \rightarrow \infty, n \in \Sigma} G^{n-1}(\alpha)=0 .
$$

As before, for $k>1, D_{\varepsilon_{n}}^{(k)}$ assumes only the values $q_{n} \varepsilon_{n}, q_{\varepsilon_{n}} \varepsilon_{n}$ and $\left(q_{\varepsilon_{n}}+q_{n}\right) \varepsilon_{n}$, assumed respectively on $W_{n}=W_{\varepsilon_{n}}, K_{n}=K_{\varepsilon_{n}}$ and $V_{n}=V_{\varepsilon_{n}}$, where we are taking $\gamma_{\varepsilon_{n}}=\varepsilon_{n}$. Note that in this case $q_{n} \varepsilon_{n} \rightarrow c$ and $q_{\varepsilon_{n}} \varepsilon_{n} \rightarrow \infty$. Note also that (18) and (19) still hold for $n \in \Sigma$. Since for fixed $k>1$ and for sufficiently large $n \in \Sigma$ we have $U^{-k+1}\left(T^{-1}\left(I_{n}\right)\right) \cap T^{-1}\left(I_{n}\right)=\varnothing$, and also $q_{\varepsilon_{n}}\left|I_{n}\right| \rightarrow 1-c$, we conclude that multiplying and dividing by $\varepsilon_{n}$ the right hand side of (18) and (19) give

$$
\begin{aligned}
& \mu\left\{\omega \in S^{1}: D_{\varepsilon_{n}}^{(k)}(\omega)=q_{n} \varepsilon_{n}\right\} \rightarrow c+(1-c)=1 \text { and } \\
& \mu\left\{\omega \in S^{1}: D_{\varepsilon_{n}}^{(k)}(\omega)=q_{\varepsilon_{n}} \varepsilon_{n}\right\} \rightarrow 0 .
\end{aligned}
$$

We conclude then that $F_{\varepsilon_{n}}^{(k)}(t)$ converges in law for every $k>0$. Now we study the convergence of $F_{\varepsilon_{n}}^{\left(k_{1}, \cdots, k_{r}\right)}\left(t_{1}, \cdots, t_{r}\right)$, where $1<k_{1}<\cdots<k_{r}$. As before we introduce the indices $\ell_{n, 1}=q_{n} \varepsilon_{n} \rightarrow c$ and $\ell_{n, 2}=q_{\varepsilon_{n}} \varepsilon_{n} \rightarrow \infty$. Put $Z_{n, 1}=W_{n}$ and $Z_{n, 2}=K_{n}$. Note that (23) still holds and when $n$ diverges we obtain

$$
\mu\left\{\omega \in S^{1}: D_{\varepsilon_{n}}^{\left(k_{s}\right)}(\omega)=\ell_{n, i_{s}}, s=1, \cdots, r\right\} \rightarrow 0
$$

if $i_{s}=2$ for some $s$, and

$$
\mu\left\{\omega \in S^{1}: D_{\varepsilon_{n}}^{\left(k_{s}\right)}(\omega)=\ell_{n, 1}, s=1, \cdots, r\right\} \rightarrow 1 .
$$

This shows that for every choice of $1<k_{1}<\cdots<k_{r}, F_{\varepsilon_{n}}^{\left(k_{1}, \cdots, k_{r}\right)}\left(t_{1}, \cdots, t_{r}\right)$ converges in law to $\lim _{n \rightarrow \infty, n \in \Sigma} \prod_{s=1}^{r} F_{\varepsilon_{n}}^{\left(k_{s}\right)}\left(t_{s}\right)$. Now, as before, for the case of $F_{\varepsilon_{n}}^{\left(1, k_{2}, \cdots, k_{r}\right)}\left(t_{1}, \cdots, t_{r}\right)$ we study the convergence of $G_{n, i_{2}, \cdots, i_{r}}^{\left(1, k_{2}, \cdots, k_{r}\right)}(t)$ of (24). Suppose $\varepsilon_{n} k \leq t<\varepsilon_{n}(k+1)$. For $k \leq q_{n}$ we have

$$
G_{n, i_{2}, \cdots, i_{r}}^{\left(1, k_{2}, \cdots, k_{r}\right)}(t)=k \mu\left(\bigcap_{s=2}^{r} U^{-k_{s}+1}\left(Z_{n, i_{s}}\right)\right),
$$

and hence if $i_{s}=2$ for some $s>1$ then $G_{n, i_{2}, \cdots, i_{r}}^{\left(1, k_{2}, \cdots, k_{r}\right)}(t) \rightarrow 0$ for $t<c$. Otherwise we have $G_{n, 1, \cdots, 1}^{\left(1, k_{2}, \cdots, k_{r}\right)}(t) \rightarrow t$ for $t<c$. For $q_{n}<k \leq q_{\varepsilon_{n}}$ we have

$$
\begin{aligned}
G_{n, i_{2}, \cdots, i_{r}}^{\left(1, k_{2}, \cdots, k_{r}\right)}(t)= & q_{n} \mu\left(\bigcap_{s=2}^{r} U^{-k_{s}+1}\left(Z_{n, i_{s}}\right)\right) \\
& +\left(k-q_{n}\right) \mu\left(\bigcap_{s=2}^{r} U^{-k_{s}+1}\left(Z_{n, i_{s}}\right) \cap T^{-1}\left(I_{n}\right)\right),
\end{aligned}
$$

and then if $i_{s}=2$ for some $s>1$, we obtain $G_{n, i_{2}, \cdots, i_{r}}^{\left(1, k_{2}, \cdots, k_{r}\right)}(t) \rightarrow 0$ for $t \geq c$. Otherwise we have $G_{n, 1, \cdots, 1}^{\left(1, k_{2}, \cdots, k_{r}\right)}(t) \rightarrow c$ for $t \geq c$. This shows that for every choice of $0<k_{1}<$ $\cdots<k_{r}, F_{\varepsilon_{n}}^{\left(k_{1}, \cdots, k_{r}\right)}\left(t_{1}, \cdots, t_{r}\right)$ converges in law to $\lim _{n \rightarrow \infty, n \in \Sigma} \prod_{s=1}^{r} F_{\varepsilon_{n}}^{\left(k_{s}\right)}\left(t_{s}\right)$. 
The above computations show that the point process $\mathcal{T}_{\varepsilon_{n}}$ with $\gamma_{\varepsilon_{n}}=\varepsilon_{n}$ (under the subsequence $n \in \Sigma$ ) converges in law to a stationary and independent limit point process. Moreover, the limit point process is with probability $c$ the so-called lattice point process with increment $c$ and it is a degenerate process with probability $1-c$.

Now we deal with the case of $\gamma_{\varepsilon_{n}}=\left|I_{n}\right|$. For $k>1, D_{\varepsilon_{n}}^{(k)}$ assumes only the values $q_{n}\left|I_{n}\right|, q_{\varepsilon_{n}}\left|I_{n}\right|$ and $\left(q_{\varepsilon_{n}}+q_{n}\right)\left|I_{n}\right|$, assumed respectively on $W_{n}=W_{\varepsilon_{n}}, K_{n}=K_{\varepsilon_{n}}$ and $V_{n}=V_{\varepsilon_{n}}$. Note that $q_{n}\left|I_{n}\right| \rightarrow 0$ and $q_{\varepsilon_{n}}\left|I_{n}\right| \rightarrow 1-c$. Note also that (18) and (19) still hold with $\varepsilon_{n}$ replaced by $\left|I_{n}\right|$. Therefore we obtain as in (25)

$$
\begin{aligned}
& \mu\left\{\omega \in S^{1}: D_{\varepsilon_{n}}^{(k)}(\omega)=q_{n}\left|I_{n}\right|\right\} \rightarrow c+(1-c)=1 \text { and } \\
& \mu\left\{\omega \in S^{1}: D_{\varepsilon_{n}}^{(k)}(\omega)=q_{\varepsilon_{n}}\left|I_{n}\right|\right\} \rightarrow 0 .
\end{aligned}
$$

Hence $F_{\varepsilon_{n}}^{(k)}(t)$ converges in law for every $k>0$. Consider $F_{\varepsilon_{n}}^{\left(k_{1}, \cdots, k_{r}\right)}\left(t_{1}, \cdots, t_{r}\right)$, where $1<k_{1}<\cdots<k_{r}$. Introduce the indices $\ell_{n, 1}=q_{n}\left|I_{n}\right| \rightarrow 0$ and $\ell_{n, 2}=$ $q_{\varepsilon_{n}}\left|I_{n}\right| \rightarrow 1-c$. Put $Z_{n, 1}=W_{n}$ and $Z_{n, 2}=K_{n}$. From (23), which still holds in this case, we obtain

$$
\mu\left\{\omega \in S^{1}: D_{\varepsilon_{n}}^{\left(k_{s}\right)}(\omega)=\ell_{n, i_{s}}, s=1, \cdots, r\right\} \rightarrow 0
$$

if $i_{s}=2$ for some $s$, and

$$
\mu\left\{\omega \in S^{1}: D_{\varepsilon_{n}}^{\left(k_{s}\right)}(\omega)=\ell_{n, 1}, s=1, \cdots, r\right\} \rightarrow 1 .
$$

This shows that for every choice of $1<k_{1}<\cdots<k_{r}, F_{\varepsilon_{n}}^{\left(k_{1}, \cdots, k_{r}\right)}\left(t_{1}, \cdots, t_{r}\right)$ converges in law to $\lim _{n \rightarrow \infty, n \in \Sigma} \prod_{s=1}^{r} F_{\varepsilon_{n}}^{\left(k_{s}\right)}\left(t_{s}\right)$. For the case of $F_{\varepsilon_{n}}^{\left(1, k_{2}, \cdots, k_{r}\right)}\left(t_{1}, \cdots, t_{r}\right)$ we study as before the convergence of $G_{n, i_{2}, \cdots, i_{r}}^{\left(1, k_{2}, \cdots, k_{r}\right)}(t)$ of (24). Suppose $\left|I_{n}\right| k \leq t<$ $\left|I_{n}\right|(k+1)$. For $q_{n}<k \leq q_{\varepsilon_{n}}$ we have

$$
\begin{aligned}
G_{n, i_{2}, \cdots, i_{r}}^{\left(1, k_{2}, \cdots, k_{r}\right)}(t)= & q_{n} \mu\left(\bigcap_{s=2}^{r} U^{-k_{s}+1}\left(Z_{n, i_{s}}\right)\right) \\
& +\left(k-q_{n}\right) \mu\left(\bigcap_{s=2}^{r} U^{-k_{s}+1}\left(Z_{n, i_{s}}\right) \cap T^{-1}\left(I_{n}\right)\right),
\end{aligned}
$$

and then if $i_{s}=2$ for some $s>1$, we obtain $G_{n, i_{2}, \cdots, i_{r}}^{\left(1, k_{2}, \cdots, k_{r}\right)}(t) \rightarrow 0$ for $0<t \leq 1-c$. Otherwise we have $G_{n, 1, \cdots, 1}^{\left(1, k_{2}, \cdots, k_{r}\right)}(t) \rightarrow c+t$ for $0<t \leq 1-c$. This shows that for every choice of $0<k_{1}<\cdots<k_{r}, F_{\varepsilon_{n}}^{\left(k_{1}, \cdots, k_{r}\right)}\left(t_{1}, \cdots, t_{r}\right)$ converges in law to $\lim _{n \rightarrow \infty, n \in \Sigma} \prod_{s=1}^{r} F_{\varepsilon_{n}}^{\left(k_{s}\right)}\left(t_{s}\right)$.

The above computations show that the point process $\mathcal{T}_{\varepsilon_{n}}$ with $\gamma_{\varepsilon_{n}}=\left|I_{n}\right|$ (under the subsequence $n \in \Sigma$ ) converges in law to a non-stationary but independent limit point process. However, in this case, the limit point process has no increment with probability one and the first hit is a mixed random variable with distribution giving probability $c$ to the origin and uniform distribution in the interval $[0,1-c]$ with probability $1-c$.

\section{The RETURN TIME POINT PROCESS}

Conditioning the point process $\mathcal{T}_{\varepsilon}$ to $\mathfrak{I}_{\varepsilon}$ we obtain the return time point process $\mathcal{T}_{\varepsilon}^{r}$. To study convergence in law we consider as before the sequence of random 
variables $X_{\varepsilon}^{(k)}$, and defining $D_{\varepsilon}^{(k)}=X_{\varepsilon}^{(k)}-X_{\varepsilon}^{(k-1)}$ we study the convergence of the joint distribution functions

$$
F_{\varepsilon}^{\left(k_{1}, \cdots, k_{r}\right)}\left(t_{1}, \cdots, t_{r}\right)=\varepsilon^{-1} \mu\left\{\omega \in \mathfrak{I}_{\varepsilon}: D_{\varepsilon}^{\left(k_{s}\right)}(\omega) \leq t_{s}, s=1, \cdots, r\right\},
$$

for $0<k_{1}<\cdots<k_{r}$. Suppose the time scale is given by $\gamma_{\varepsilon}$. Then the random variables $T_{\varepsilon}^{k}$ assume only the values $q_{n} \gamma_{\varepsilon}, q_{\varepsilon} \gamma_{\varepsilon}$ or $\left(q_{\varepsilon}+q_{n}\right) \gamma_{\varepsilon}$, respectively on $W_{\varepsilon}, K_{\varepsilon}$ and $V_{\varepsilon}$. Here $n$ is the unique integer such that $\left|I_{n}\right|<\varepsilon \leq\left|I_{n-1}\right|$ and we are assuming $f$ is the rigid rotation by $\alpha$. For a subsequence $\varepsilon_{n} \rightarrow 0$ consider $\ell_{n, 1}=q_{n} \gamma_{\varepsilon_{n}}$ and $\ell_{n, 2}=q_{\varepsilon_{n}} \gamma_{\varepsilon_{n}}$. Define as in the previous section $Z_{n, 1}=W_{n}=W_{\varepsilon_{n}}$ and $Z_{n, 2}=K_{n}=K_{\varepsilon_{n}}$. From (22) we have for $0<k_{1}<\cdots<k_{r}$,

$$
\varepsilon_{n}^{-1} \mu\left\{\omega \in \mathfrak{I}_{\varepsilon_{n}}: D_{\varepsilon_{n}}^{\left(k_{s}\right)}(\omega)=\ell_{n, i_{s}}, s=1, \cdots, r\right\}=\varepsilon_{n}^{-1} \mu\left(\bigcap_{s=1}^{r} U^{-k_{s}+1}\left(Z_{n, i_{s}}\right)\right),
$$

for any choice of $i_{s} \in\{1,2\}, s=1, \cdots, r$. Suppose $\varepsilon_{n}=c\left|I_{n-1}\right|$ for some fixed $0<c<1$ and consider $\gamma_{\varepsilon_{n}}=\varepsilon_{n}$. Convergence under a subsequence occurs exactly in the cases (a) or (b) of Proposition [1].

In the case (b), we take $c>\theta$, and we have

$$
\varepsilon_{n}^{-1} \mu\left\{\omega \in \mathfrak{I}_{\varepsilon_{n}}: D_{\varepsilon_{n}}^{\left(k_{s}\right)}(\omega)=\ell_{n, i_{s}}, s=1, \cdots, r\right\} \rightarrow \mu\left(\bigcap_{s=1}^{r} U_{c, \theta}^{-k_{s}+1}\left(Z_{i_{s}}\right)\right),
$$

where we have defined $Z_{1}=W$ and $Z_{2}=K$ as in the previous section, and we note that $\ell_{n, i} \rightarrow d_{i}$. It is interesting to note that the asymptotic distributions of $D_{\varepsilon_{n}}^{(k)}(\omega)$ for $\omega \in S^{1}$ with $k>1$ are different from the asymptotic distributions of $D_{\varepsilon_{n}}^{(k)}(\omega)$ conditionally to $\mathfrak{I}_{\varepsilon_{n}}$. Taking $\gamma_{\varepsilon_{n}}=\left|I_{n}\right|$ changes the asymptotic distributions only in that the time scale is multiplied by a factor $\theta / c$.

Now in the case (a) of Proposition [1] and $\gamma_{\varepsilon_{n}}=\varepsilon_{n}$, we have $\ell_{n, 1} \rightarrow c$ and $\ell_{n, 2} \rightarrow \infty$. From (27) we obtain

$$
\varepsilon_{n}^{-1} \mu\left\{\omega \in \mathfrak{I}_{\varepsilon_{n}}: D_{\varepsilon_{n}}^{\left(k_{s}\right)}(\omega)=\ell_{n, i_{s}}, s=1, \cdots, r\right\} \rightarrow 0
$$

if $i_{s}=2$ for some $s$, and

$$
\varepsilon_{n}^{-1} \mu\left\{\omega \in \mathfrak{I}_{\varepsilon_{n}}: D_{\varepsilon_{n}}^{\left(k_{s}\right)}(\omega)=\ell_{n, 1}, s=1, \cdots, r\right\} \rightarrow 1 .
$$

Therefore in this case $\mathcal{T}_{\varepsilon_{n}}^{r}$ converges in law to a deterministic point process with point masses at $c \mathbb{N}$. Finally, for $\gamma_{\varepsilon_{n}}=\left|I_{n}\right|$, we have $\ell_{n, 1} \rightarrow 0$ and $\ell_{n, 2} \rightarrow 1-c$. From (27) again we obtain (28) and (29). Therefore in this case $\mathcal{T}_{\varepsilon_{n}}^{r}$ converges in law to the trivial point process with point mass at the origin.

This shows that there is no loss of tightness for return times and there is no coexistence of non-trivial limit point processes by choosing different scales in the case of return times.

\section{REFERENCES}

[Bre] L. Breiman, Probability theory, Addison-Wesley Series in Statistics, 1968. MR 37:4841

[BZ] X. Bressaud \& R. Zweimüller, Non exponential law of entrance times in asymptotically rare events for intermittent maps with infinite invariant measure, Annales Henri Poincaré 2, No.3 (2001), 501-512. MR 2002f:37018

[Coe] Z. Coelho, Asymptotic laws for symbolic dynamical systems, in Topics in Symbolic Dynamics and Applications, Eds. F. Blanchard, A. Maass \& A. Nogueira, LMS Lecture Notes Series 279, Cambridge Univ. Press, (2000) 123-165. MR 2001h:37016 
[CC] Z. Coelho \& P. Collet, Limit law for the close approach of two trajectories of expanding maps of the circle, Prob. Th. and rel. fields 99 (1994), 237-250. MR 95g:60034

[CF] Z. Coelho \& E. de Faria, Limit laws of entrance times for homeomorphisms of the circle, Israel J. Math. 93 (1996), 93-112. MR 97e:58141

[CG] P. Collet \& A. Galves, Asymptotic distribution of entrance times for expanding maps of the interval, Dynamical Systems and Applications 139-152, World Sci. Ser. Appl. Anal., 4, World Sci. Publishing, 1995. MR 97b:58083

[DM] F. Durand \& A. Maass, Limit laws of entrance times for low complexity Cantor minimal systems, Nonlinearity 14, No.4 (2001), 683-700. MR 2002f:37001

[DV] D.J. Daley \& D. Vere-Jones, An introduction to the theor y of point processes, Springer Series in Statistics, Springer-Verlag, 1988. MR 90e:60060

[FHZ1] S. Ferenczi, C. Holton \& L. Zamboni, Structure of three-interval exchange transformations I: an arithmetic study, Ann. Inst. Fourier 51, 4 (2001), 861-901. MR 2002g:11116

[FHZ2] S. Ferenczi, C. Holton \& L. Zamboni, Combinatorics of three-interval exchanges, Proceedings ICALP 2001, Lecture Notes in Computer Science 2076 (2001), Springer-Verlag, $567-578$.

[FHZ3] S. Ferenczi, C. Holton \& L. Zamboni, Structure of three-interval exchange transformations II: combinatorial description of the trajectories, J. Analyse Math 89 (2003), 239-276.

[FHZ4] S. Ferenczi, C. Holton \& L. Zamboni, Structure of three-interval exchange transformations III: ergodic and spectral properties, submitted.

[FHZ5] S. Ferenczi, C. Holton \& L. Zamboni, Joinings of three-interval exchange transformations, submitted.

[GS] G.R. Grimmett \& D.R. Stirzaker, Probability and Random Processes, Clarendon Press, Oxford, 1992. MR 93m:60002

[Hir] M. Hirata, Poisson law for Axiom A diffeomorphisms, Erg. Th. Dyn. Sys 13 (1993), 533-556. MR 94m:58137

[HSV] M. Hirata, B. Saussol \& S. Vaienti, Statistics of return times: a general framework and new applications, Comm. Math. Physics 206 (1999), 33-55. MR 2001c:37007

[Nev] J. Neveu, Processus pontuels, in Springer Lecture Notes in Maths 598 (1976), 249-445. MR 57:14132

[Pit] B. Pitskel, Poisson limit law for Markov chains, Erg. Th. Dyn. Sys. 11 (1991), 501-513. MR 92j:60081

Department of Mathematics, University of York, Heslington, York YO10 5DD, UNITED KingDOM

E-mail address: zc3@york.ac.uk 